\title{
Sick Societies: Responding to the Global Challenge of Chronic Disease
}

\author{
edited by David Stuckler and Karen Siegel \\ New York: Oxford University Press 2011 \\ ISBN 978-0-19957-440-7 \\ Softcover, $\$ 75,352 \mathrm{pp}$.
}

\author{
Reviewed by Raghubar D. Sharma \\ Ontario Ministry of Children and Youth Services
}

The first globalization took place between the 16th and the 18th centuries as a result of improved maritime technology. During this period, as European powers colonized the Americas, Africa, and Asia, the diseases of Europe such as syphilis, smallpox, and plague travelled to the colonies, resulting in a massive scale of morbidity and mortality among the native populations. The second major globalization is now underway, due to advances in information technology, and many countries are experiencing an unprecedented rise in chronic diseases. These diseases are not only causing misery and early mortality in the world, but are also bringing an unbearable social and economic burden to societies that are unprepared for this challenge. There is urgency for understanding changes in disease patterns across the globe, and this book is a valuable addition to the knowledge of undergoing changes in morbidity.

The change from infectious diseases to chronic diseases in a society has been used as an indicator of development. As societies develop, the incidence of communicable diseases decreases and that of the chronic diseases increases. The book shows that this relationship between infectious diseases and chronic diseases is no longer tenable, and the incidence of chronic diseases is on the rise everywhere. This finding is a major contribution of the book and a timely alert to policymakers.

This book consists of eight chapters. Chapter 1 provides a basic understanding of the major chronic diseases that are major killers-cardiovascular diseases, cancers, respiratory diseases, and diabetes. The chapter explains how four major factors - tobacco, unhealthy diet, inactivity, and alcohol—threaten the lives of people around the globe by increasing the incidence of the major chronic diseases.

Chapter 2 demonstrates how industry marketing of products related to the risk factors of chronic diseases makes unhealthy choices more economical for the consumer. The strategies followed by the food industry to market products related to the risk factors of chronic diseases include low prices, easier access, and smart marketing techniques. The chapter concludes with three country case studies: (1) Russia's free-market policies cause more than 3 million deaths related to heart disease and alcohol; (2) crashing economies in Japan, Finland, and Cuba force their populaces to return to traditional healthy eating, resulting in the reduction of chronic diseases; and (3) the elimination of traditional living in Nauru by an unsustainable development model.

Chapter 3 is devoted to the social and economic costs of chronic diseases. It shows that chronic diseases are costly not only due to healthcare expenditures but also in terms of the labour market: the diseased are more likely to be low-earners or unemployed. The chapter demonstrates how chronic diseases can trap a family in intergenerational poverty. It also makes a case for government intervention and discusses the consequences of intervention.

The first part of Chapter 4 deals with the management of chronic diseases, and the second part discusses prevention strategies. It argues that health management systems set up to deal with infectious diseases do not work for the management of chronic diseases. It also outlines barriers to achieving transformation of medical 
care, and the potential benefits of pharmaceutical development to human development. The second part of the chapter reveals a gap between medical interventions and preventative approaches. Finally, the chapter recommends a community-based model as an alternative to the current models, in which scientists provide costbenefit analyses and suggest solutions.

Chapter 5 draws attention to inequities in healthcare funding for chronic diseases. It also provides an analysis of the conflict of interest for private donors due to their relationship with the pharmaceutical and food industries. The second part of the chapter proposes creation of a social movement that would be based on sociological models and would utilize best practices for the environment, HIV/AIDS, and civil rights programs. It proposes strategies to address funding inequalities.

In Chapter 6, the authors invite contributors from PepsiCo's Global Health and Wellness Strategy to emphasize that food industry investment in health is not only socially responsible but is also highly profitable. The chapter argues that it is in the interest of the food industry to create partnerships with governments and public health agencies to improve population health. The second part of the chapter presents a critique of the food industry by drawing analogies to the tactics used by the tobacco industry that undermined public health research and effective interventions. At the end of the chapter, a series of recommendations is presented to avoid conflicts of interest with government and to win public confidence.

Chapter 7 presents case studies from the rapidly growing economies of Brazil, China, India, Mexico, and South Africa. These case studies elucidate that these countries are still using public health strategies akin to those for controlling infectious diseases. Though these countries are very different from each other, they have similar inequitable rises in chronic diseases, which threaten their economic growth.

Chapter 8 provides a series of suggestions as to how citizens can help for a healthier world by building networks, reframing debate, transforming the private sector, and supporting litigation and human rights groups.

The book uses acronyms profusely, leaving the reader bewildered. Because there are an inordinate number of acronyms used in this book, at least an appendix with an alphabetical list of the full forms of these acronyms would be highly desirable. Similarly, some tabular analyses are also confusing. For example, regarding Table 7.2, the percentage increase is discussed in the text but is not given in the table. Finally, some material is repetitive, particularly in the first three chapters.

Needless to say, the book is highly ambitious and is an important contribution to the literature not only of chronic diseases but also medical sociology and the discipline of public health. I would highly recommend this book to anyone interested in chronic diseases, social and economic development, and the globalization of public health. The book provides a lens on the impact of social and economic changes being ushered by the globalization. The authors have succeeded in alerting informed readers and policymakers about the impending threat of human misery, and they must be congratulated. 